\title{
Desarrollo de elementos para un sistema NWP-NWC
}

https://doi.org/10.31978/639-19-010-0.317

\author{
Carlos Geijo Guerrero' (cgeijog@aemet.es)
}

${ }^{1}$ AEMET / Departamento de Desarrollo y Aplicaciones / Área de Modelización

\begin{abstract}
RESUMEN
La aplicación de modelos de predicción numérica en área limitada (NWP-LAM) a fenómenos meteorológicos con tiempos de predecibilidad cortos (i.e. unas pocas horas) parece haber ido ganando presencia en los últimos tiempos entre los operadores de este tipo de sistemas, tanto en Europa como más allá. Este rango de predicciones, a veces conocida como «NowCasting (NWC)» 0 «Short-Range NWP» 0 «Very Short-Range NWP», constituye un segmento de productos en los que sistemas NWP enfocados a la predicción a escala global difícilmente podrán entrar algún día, no importa cuán fina llegue a ser la discretización empleada por ellos. La razón fundamental para respaldar esta previsión reside en la incompatibilidad entre las técnicas de asimilación de datos (y estrategias de observación) que resultan ser eficaces en uno y otro ámbito. La necesidad ineludible de utilizar ciclos de actualización muy frecuentes que puedan capturar dinámicas atmosféricas con tiempos de vida de unas horas, y todos los ajustes técnicos que de este requisito fundamental se derivan, proyecta sobre los sistemas de NWP-Global una luz en la cual estos sistemas aparecen como maquinarias excesivamente rígidas, claramente inadecuadas para este fin.

En el pasado se han desplegado muchas aplicaciones NWC integradas en la cadena de post-proceso de sistemas NWP que han demostrado cierta utilidad en la extrapolación inmediata de observaciones recogidas en modo casi continuo como radares o satélites. El concepto NWP-NWC persigue una superación de esta práctica mediante una integración en tiempo casi real de este flujo de observaciones en la inicialización del modelo.

En esta ponencia se presentarán algunos desarrollos llevados a cabo en AEMET persiguiendo el objetivo de implementar un prototipo de sistema NWP-NWC a partir de adaptaciones e innovaciones sobre el sistema NWP-LAM conocido como HARMONIE-AROME. Por ejemplo, extracción de pseudo-observaciones por alineamiento de campos del modelo con imágenes de radar y asimilación sincronizada al paso de tiempo del modelo mediante técnicas variacionales.
\end{abstract}

PALABRAS CLAVE: predicción numérica; nowcasting; asimilación de datos; corrección de errores de posición; inicialización; ligaduras variacionales.

\section{INTRODUCCIÓN}

El conjunto de técnicas recogidas bajo la denominación NWP-NWC persiguen mejorar las predicciones a corto y muy corto plazo generadas mediante sistemas NWP-LAM, en otras palabras, mejorar la predicción de fenómenos meteorológicos intrínsicamente menos predecibles, y hacerlo sin perder de vista el paradigma determinista no caótico (precisión y exactitud). Como muestra la experiencia con productos NWC, estas predicciones son potencialmente útiles en la gestión de situaciones de riesgo (e.g. incendios forestales). La dirección a seguir apunta hacia la integración de elementos propios de NWC (observaciones y productos) en el flujo productivo de generación de análisis y predicciones objetivas mediante sistemas NWP-LAM, incorporando lo mejor de ambas técnicas: adaptabilidad y eficiencia por un lado y complejas representaciones algorítmicas de procesos dinámicos y físicos de la atmósfera por otro. 
Algunas de las razones por las cuales NWP-LAM encuentra dificultades en su utilización en el ámbito de la predicción a muy corto plazo tienen su origen en los procesos de asimilación de observaciones e inicialización de los modelos. Las estrategias de asimilación actualmente empleadas en NWP-LAM estan condicionadas por un legado procedente de la predicción numérica a escala global que le resta adaptabilidad. La introducción de observaciones incongruentes con el estado del modelo generado por sus esquemas numéricos tiene efectos disruptivos que limitan su aplicabilidad en el muy corto plazo. A continuación se presentan dos técnicas que abordan estas dificultades.

La primera es un método para la correción de errores de posición mediante la deformación continua de campos del modelo hasta hacerlos coincidir con una referencia (u observación) dada, método conocido como Field Alignment (FA). El objetivo de esta breve comunicación es presentar el método FA independientemente de cuál sea el tipo de observación que se utiliza, si bien es cierto que su implementación no es en absoluto independiente de las características específicas de aquel. El ejemplo con imágenes radar es aquí idóneo dado el uso intensivo del dato radar en procedimientos NWC.

La segunda técnica es un algoritmo de inicialización desarrollado a partir de la consideración de la dinámica semiimplícita de HARMONIE-AROME (conocida como ALADIN-NH) como expresión precisa de los balances dinámicos deseables en unas condiciones iniciales para predicciones efectuadas con HARMONIEAROME. Se conoce como Variational Constraints (VC). VC tiene características de algoritmos tipo nudging que lo hacen útil para sistemas de asimilación de datos en tiempo casi real, si bien aún no ha sido testado en este contexto. Aquí se mencionan pruebas llevadas a cabo sobre análisis de viento generados con el método FA utilizando imágenes y pseudo-imágenes radar de viento radial Doppler, sobre análisis obtenidos mediante el algoritmo basado en ensembles LETKF, y sobre análisis ordinarios 3D-VAR en modo univariante.

\section{CORRECCIÓN DE ERRORES DE POSICIÓN MEDIANTE FIELD ALIGNMENT}

RAVELA et al. (2007) distinguen dos tipos de errores en una predicción, errores de amplitud y errores de posición. Los autores subrayan la importancia de estos últimos en la predicción de fenómenos adversos, intensos y localizados y argumentan que precisan de un tratamiento específico que métodos clásicos de asimilación (e. g. 3D-VAR), eficaces en la corrección de errores de amplitud, no pueden proporcionan. Estos métodos suelen producir en este tipo de situaciones «análisis deformados» que constituyen en sí mismos otra fuente de errores para predicciones sucesivas (carácter autorregresivo del sistema asimilación-predición). El empleo de ensembles para estimar los errores del modelo en una forma «dependiente del flujo» no mejora significativamente la situación porque los errores de posición introducen sesgos que invalidan los supuestos de la estimación, lo cual conduce eventualmente a la aparición de estos mismos efectos distorsionantes en análisis obtenidos a partir de ensembles.

La solución propuesta consiste en una (re)formulación bayesiana del problema del análisis en la cual la inferencia de errores de amplitud y posición está condicionada a unos «a prioris» construidos a partir de predicciones y de ligaduras al campo de deformación que corrige los errores de posición. De esta forma son capaces de definir un «objetivo» $\mathrm{o}$ «función de coste» cuyo extremo minimiza conjuntamente errores de amplitud y de posición. En la versión más simple de su algoritmo, denominada versión secuencial o two-step algorithm, el campo de deformación q viene dado por la solución de una ecuación en derivadas parciales (eq. 26 en RAVELA et al. (2007)). Esta ecuación puede resolverse de manera eficiente (se trata de una ecuación no lineal que requiere de procedimientos iterativos) trabajando en el espacio de números de onda y en un dominio extendido que permite solventar el problema que plantea su singularidad en $\mathbf{k}=0$ (GEIJO, 2011).

\subsection{Empleo del método en imágenes radar de viento radial Doppler}

Esta técnica ha sido testada con imágenes radar en varios experimentos. La implementación del método no es en absoluto independiente del tipo de dato utilizado, por lo que se requieren soluciones ad hoc en cada caso. 
Por ejemplo, cuando se utilizan imágenes de viento radial Doppler (GEIJo, 2013) es preciso considerar la existencia en la imagen de zonas sin precipitación que no proporcionan medida de viento. El procedimiento adoptado para soslayar esta dificultad consiste en detectar y clasificar estas zonas como «interiores» $\mathrm{O}$ «externas» (GEIJo, 2013, t17). Para las primeras el término de forzamiento en la ecuación de FA no está sujeto a restricción alguna, y el campo de deformación puede propagarse de forma continua a su interior. En el caso de las segundas, en cambio, se impone la condición de contorno de campo de deformación nulo $(\mathbf{q}=0)$. Las (afortunadamente) pequeñas interferencias con la orografía son tratadas de igual manera. Otros puntos problemáticos que tuvieron que ser abordados en el curso de estos desarrollos provienen de la especificidad de la geometría de la observación mediante radar. El área iluminada por los pulsos electromagnéticos emitidos por la antena del radar tiene una forma cónica que intersecta los niveles del modelo. Estas intersecciones son anulares cuando se proyectan horizontalmente (GEIJo, 2013, t16). Hubo que investigar la conveniencia de realizar el alineamiento sobre los niveles del modelo (que están modulados por la orografía) o sobre superficies horizontales. Ambas opciones presentan ventajas e inconvenientes, pero finalmente se optó por la segunda debido a que el operador que realiza las transformaciones geométricas produce una huella mucho más suave en este caso, facilitando la eliminación de ruido en el importante término de forzamiento en la ecuación de FA.

Las primeras pruebas sistemáticas se llevaron a cabo con dato de la red de radares de AEMET correspondientes al periodo septiembre-octubre 2012, periodo que incluye la campaña SOP-1 del proyecto HyMex. La figura 1 muestra la curva de error para las seis primeras horas de predicción para el viento radial, promediado sobre unos 150 casos. Los diferentes colores corresponden a diferentes pruebas llevadas a cabo. La curva morada representa la situación downscaling que marca el «nivel 0» sin asimilación. Las curvas azul y verde dan los resultados para dos experimentos de asimilación del dato radar con 3D-VAR convencional y FA respectivamente. Como cabe esperar, el ajuste a la observación es mayor con FA, pero la pendiente de la curva lo es también, resultando en una disipación del efecto en un tiempo de unas dos horas. La curva roja corres-

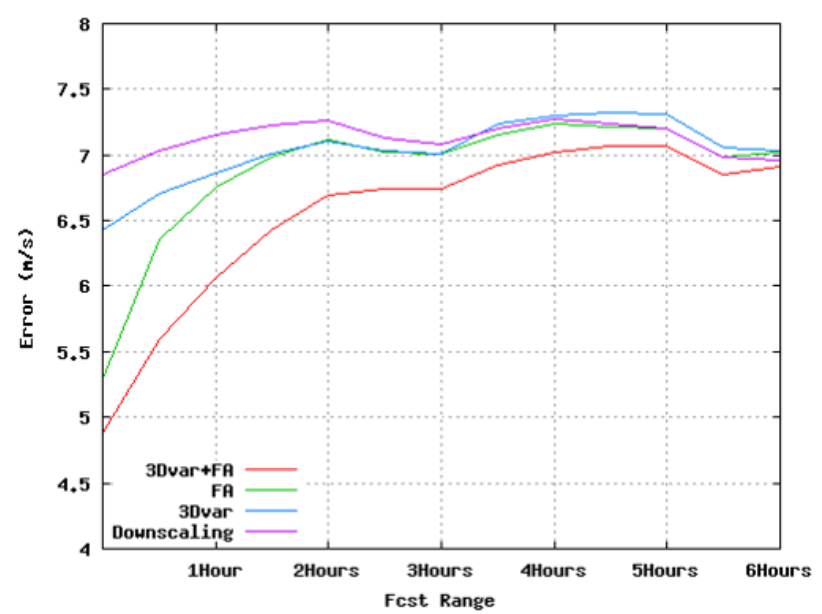

Figura 1. Curvas de crecimiento de error en el parámetro viento radial para diferentes experimentos de asimilación con dato radar usando la técnica de Field Alignment. ponde a un experimento en el que se ensayó un procedimiento híbrido consistente en una alineación del campo de viento, y el posterior proceso de las correcciones obtenidas mediante la matriz de covarianzas de errores del modelo (GEJJo, 2013, t26, t27, t28). Esta última corrección es similar a la «corrección en amplitud» propuesta por RAVELA et al. (2007) como «segundo paso» en su algorimo secuencial, pero difiere en algunos detalles.

\subsection{Empleo del método FA con imágenes radar de reflectividad $Z$}

La técnica FA también puede emplearse en la asimilación de reflectividades radar. En principio la situación es más sencilla que en el caso del dato de viento radial, dado que en una imagen radar de reflectividad no hay zonas desprovistas de datos. Sin embargo surgen otras dificultades específicas a esta aplicación. En primer lugar debe realizarse un chequeo preliminar de semejanza entre el campo de precipitación en el modelo y la imagen de radar con la cual se va a alinear, puesto que es claro que el procedimiento de corrección de errores de posición no debe generar o eliminar precipitación por sí mismo. Satisfecha esta primera condición, el algoritmo funciona correctamente, si bien el número de situaciones en que es aplicable puede quedar significativamente reducido. También existen dificultades adicionales relativas a la conexión física entre reflectividad y las variables de control en el análisis (e. g. humedad del aire). En algunos procedimientos de asimilación operativos actuales se adopta la solución de utilizar el dato de reflectividad como un proxy para generar 
pseudo-observaciones de humedad relativa del aire. En los experimentos con FA se procede de otra forma. El alineamiento del campo $Z$ (o $\log Z$ ) entre observación y modelo produce un campo de deformación que es utilizado para corregir la posición en los campos de humedad específica $q$, temperatura $T$ e hidrometeoros $\left\{q_{i}\right\}$. Dado el carácter granular de algunos de estos campos, es preciso utilizar esquemas de interpolación más precisos que una mera interpolación lineal o bicúbica.

A pesar de estas dificultades, se ha explorado el potencial del método FA en la asimilación de dato de reflectividad radar. Para evitar problemas añadidos derivados de eventuales caídas en la calidad de este dato (ecos espúreos, etc.), se utilizaron imágenes sintéticas o pseudo-imágenes, son pues experimentos llevados a cabo en condiciones idealizadas. La preparación de las pruebas consiste en realizar una primera simulación que es utilizada para producir observaciones sintéticas y también como referencia de validación. Otras simulaciones pueden incorporar estas pseudo-observaciones en diferentes modos, o bien ser privadas completamente de esta información. Sus predicciones son después utilizadas para medir la magnitud del efecto producido. La figura 2 presenta algunos resultados obtenidos de esta manera. En la columna de la izquierda tenemos el parámetro POD (probabilidad de detección) para un amplio rango de intensidades de precipitación y para diferentes alcances de la predicción: primeras 3 horas en la fila superior, 3-6 horas en la intermedia y 6-9 horas en la inferior. La columna de la derecha muestra en el mismo formato el parámetro FAR (fracción de falsas alarmas). Estos parámetros, POD y FAR, están calculados en un área de unos $50 \times 50$ puntos de rejilla (aprox. $125 \times 125 \mathrm{~km}^{2}$ ) alrededor de la ubicación de los diferentes radares de la red AEMET considerados en estos ensayos. Se pueden ver cuatro curvas de diferentes colores que corresponden a cuatro pruebas diferentes. En rojo está la referencia o «nivel 0» que señala el experimento sin asimilación de reflectividad. La curva «twin3c» corresponde a un experimento con un ciclo de asimilación de tres horas, «twin 3cc» asimilación horaria, $\mathrm{y}$ «twin $3 \mathrm{ccUp}$ » asimilación horaria seguida de una «corrección en amplitud» mediante la matriz de covarianza de errores del modelo, siguiendo una pauta similar al caso de los experimentos con dato de viento presentados más arriba.
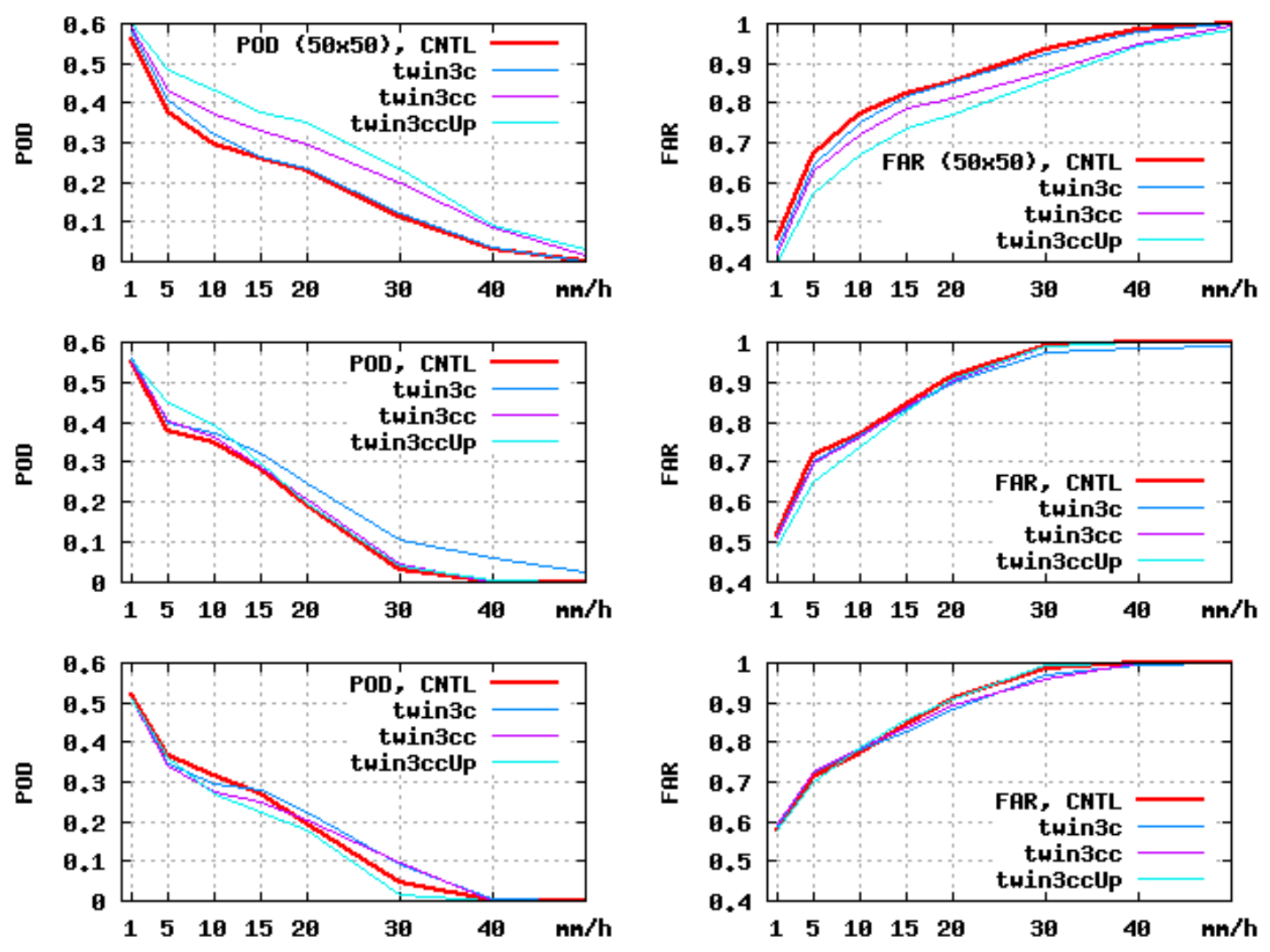

Figura 2. Validación en experimentos de asimilación de reflectividad con la técnica FA. Se presentan los parámetros POD (izquierda) y FAR (derecha) para diferentes alcances de la predicción de intensidad de precipitación. 


\section{INICIALIZACIÓN MEDIANTE LIGADURAS VARIACIONALES}

El asunto de la inicialización tiene evidente relevancia para sistemas NWP-LAM enfocados a la predicción a corto y muy corto plazo. Actualmente, la asimilación de datos en el sistema HARMONIE-AROME utiliza balances estadísticos que no incluyen su dinámica no hidrostática. Además, la intermitencia de los fenómenos mesoescalares detrae significación a las covarianzas multivariables obtenidas por estos métodos estadísticos. Estas son algunas de las motivaciones que han conducido al desarrollo de un método nuevo de inicialización mediante ligaduras variacionales (variational constraints o VC) (GEIJO y EscribÀ, 2018; GeIJo, 2018).

El punto de partida de este método es la consideración de la parte semiimplícita de la dinámica de HARMONIE-AROME (conocida como dinámica ALADIN-NH) como un enunciado preciso de qué balances dinámicos deben observarse en los análisis que serán utilizados como condiciones iniciales. Se plantea en términos de hallar el extremo de una funcional que conduce a un problema de condiciones de contorno que es resoluble mediante cuadraturas utilizando funciones de Green (GEIJo, 2018). El cálculo de estas funciones de Green pone de manifiesto una serie de propiedades que las mismas poseen, y que permiten establecer interesantes analogías entre ellas y las matrices de covarianza. Esta correspondencia puede invertirse, y considerar una matriz de covarianza como una función de Green, lo cual conduce a una técnica de modelización de covarianzas mediante integrales gaussianas. Las posibilidades de esta técnica son objeto de investigación en curso.

El algoritmo VC tiene características de esquemas tipo nudging que lo hacen adecuado para sistemas de asimilación en tiempo casi real, un requisito insoslayable para la predicción de fenómenos atmosféricos con tiempos de predecibilidad intrínseca muy breves. Esta propiedad proviene del hecho de ser la parte semiimplicita de ALADIN-NH un operador «de avance de paso de tiempo» (time-step operator). Esta posibilidad de VC no ha sido, sin embargo, testada hasta el momento. Las pruebas realizadas se han centrado en su propiedad como filtro de ruido en las condiciones iniciales, y en concreto en los contextos siguientes:

- con observaciones sintéticas obtenidas por diferencia de dos predicciones;

- con correcciones al campo de viento generadas mediante FA a partir de pseudo-imágenes radar;

- con un ensemble de análisis obtenidos mediante LETKF (Local Transform Kalman Filter, HunT et al., 2007) y observaciones convencionales;

- con análisis 3D-VAR en modo univariante y observaciones convencionales.

Los resultados de estos tests se valoran positivamente. El empleo de observaciones sintéticas permite analizar en detalle las propiedades del método. VC es capaz de generar con un nivel notable de detalle la relación «fuera de fase» entre incrementos de divergencia horizonal y vertical que exhiben estos dos parámetros en campos de diferencias entre dos predicciones a más de 12 horas, alcance para el cual los fenómenos de spin-up pueden considerarse despreciables (figura 3 en GEIJO y EscRIBÀ, 2018).

La figura 3 muestra el resultado de aplicar VC a correcciones al viento radial mediante el empleo de la técnica FA. La estructura vertical del campo de divergencia horizontal aparece filtrada (aunque la forma inclinada del haz del radar es todavía discernible), y se han generado incrementos en los campos analizados de temperatura (línea roja) y velocidad vertical (contornos blancos continuos para movimientos ascendentes y discontinuos para descendentes) mediante las relaciones dinámicas de compresibilidad y conservación del momento vertical incluidas en el sistema semiimplícito de ALADIN-NH.

Los resultados de VC como filtro para condiciones iniciales generadas a partir de LETKF pueden considerarse aceptables. VC reduce significativamente el ruido durante la primera hora de integración (figura 6 en GEIJO y ESCRIBÀ, 2018). VC también reduce significativamente el spread del ensemble a la par que no se detecta reducción en el error cuadrático medio, lo cual no es deseable. Sin embargo, cabe argumentar que no toda la 


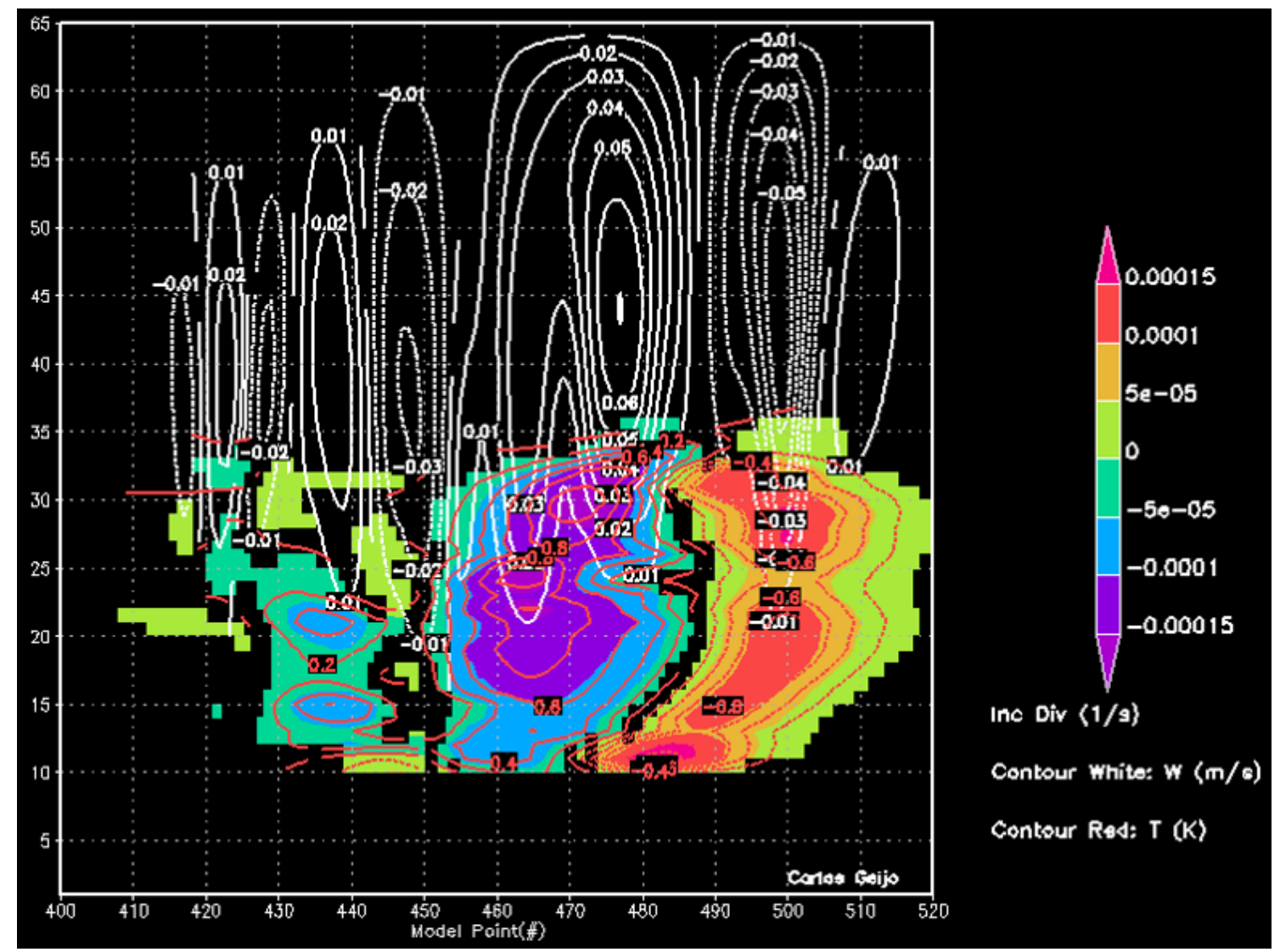

Figura 3. Balances inducidos por VC entre los campos de divergencia horizontal, velocidad vertical y temperatura a partir de correcciones al campo de viento radial Doppler mediante

la técnica de Field Alignment.

dispersión suprimida es una medida genuina de incertidumbre. Las curvas de verificación para presión en superficie y viento a 10 m (figura 7 en GEIJO y EsCRIBÀ, 2018) muestran una evolución del spread creciente con el alcance de la predicción para los experimentos con VC que está ausente en el control, un indicio de que la mayor parte de la dispersión filtrada en los análisis no es propagada por el modelo.

Finalmente los resultados con 3D-VAR en modo univariante parecen indicar la existencia de sinergias entre los balances estadísticos y VC. Los primeros incluyen un balance horizontal geostrófico y un balance hidrostático que no están considerados en VC. Las verificaciones de presión y temperatura en superficie, variables que son analizadas por VC mediante la ecuación de la tendencia y la ecuación de compresibilidad, resultan algo peores que 3D-VAR en modo multivariante. Es sin embargo fácil eliminar este efecto mediante ajustes ad hoc en parámetros de VC. La combinación de ambos VCy balances estadísticos, tiene un impacto positivo en las verificaciones de precipitación y nubosidad (figuras 8 y 9 en GEIJO y EsCRIBÀ, 2018).

\section{CONCLUSIONES}

La predicción a corto y muy corto plazo tiene interés estratégico para operadores de sistemas NWP-LAM porque los proveedores de predicciones globales difícilmente podrán llegar a cubrir con sus productos las necesidades de un amplio número de usuarios que, con carácter puntual o rutinario, encuentran utilidad en información meteorológica generada con un elevado ritmo de actualización y precisión. A partir de esta posición, es deseable mejorar los aspectos de estos sistemas NWP-LAM que, en parte por el legado recibido en sus desarrollos, dificultan la potenciación de su utilidad en este rango de predicciones y productos. 
Se presentan aquí dos desarrollos llevados a cabo en AEMET en los últimos años que pretenden mejorar uno de estos aspectos críticos, la actualización del estado del modelo mediante técnicas de asimilación. En el primer caso se trata de un algoritmo de corrección de errores de posición conocido como Field Alignment que permite extraer información a alta resolución de imágenes radar de viento y reflectividad. El segundo, conocido como ligaduras variacionales (o variational constraints, $\mathrm{VC}$ ), es un algoritmo de inicialización de las condiciones iniciales basado en la dinámica no hidrostática ALADIN-NH que utiliza el sistema NWP-LAM conocido como HARMONIE-AROME. Ambos desarrollos son complementarios pero independientes y han sido testados en diferentes experimentos con resultados prometedores, que aquí se presentan someramente. Es deseable una fase de pruebas más amplia y sistemática, si es posible incluyendo técnicas de ensembles, algo que seguramente tendrá lugar en un futuro próximo. El software que implementa sendos prototipos ha sido integrado en la versión 40 del sistema HARMONIE-AROME, lo cual debe facilitar esta tarea.

Independientemente de estos trabajos de testeo, las actividades de desarrollo de nuevas técnicas son absolutamente necesarias de cara a una efectiva integración de métodos y productos NWC en los sistemas NWP-LAM de producción de análisis y predicciones objetivas.

\section{REFERENCIAS}

Hunt, B. R. et al., 2007. Efficient Data Assimilation for Spatiotemporal Chaos: A Local Ensemble Transform Kaman Filter. Physica D., 230, 112-126 (disponible en: www.sciencedirect.com).

GeIJo, C., 2011. Data Assimilation by Field Alignment. Testing the Theory. HIRLAM Newsletter, 58, 42-51. https://www.researchgate.net/publication/281237306_Data_Assimilation_by_Field_Alignment_ Testing_the_Theory.

GeIJo, C., 2013. Assimilation of Weather Radar Data in a Convection Permitting NWP System using the Field Alignment Technique. Presentation at the 6th WMO Symposium on Data Assimilation. https:// www.researchgate.net/publication/281236886_Assimilation_of_Weather_Radar_Data_in_ a_Convection_Permitting_NWP_System_using_the_Field_Alignment_Technique.

GEIJO, C. y EsCRIBÀ, P., 2018. Variational Constraints for Data Assimilation in ALADIN-NH Dynamics. HIRLAM-ALADIN NewsLetter, 11., 13-26. https://www.researchgate.net/publication/326479446_ Variational_Constraints_for_Data_Assimilation_in_ALADIN-NH_Dynamics.

GEIJ, C., 2018. ANNEX to Variational Constraints for Data Assimilation in ALADIN-NH Dynamics. https:// www.researchgate.net/publication/327117950_ANNEX_to_Variational_Constraints_for_DA_in_ALADINNH_Dynamics.

Ravela, S. et al., 2007. Data Assimilation by Field Alignment, Physica D., 230, 127-145 (disponible en: www.sciencedirect.com ). 
